\title{
Endoscopic ultrasonography for pretreatment T-staging of gastric cancer: An in vitro accuracy and discrepancy analysis
}

\author{
YAN YAN $^{1 *}$, QI WU $^{1 *}$, ZI-YU LI ${ }^{2}$, ZHAO-DE BU $^{2}$ and JIA-FU JI ${ }^{2}$ \\ ${ }^{1}$ Endoscopy Center and ${ }^{2}$ Department of Gastrointestinal Surgery, Beijing Cancer Hospital, \\ Key Laboratory of Carcinogenesis and Translational Research (Ministry of Education), \\ Peking University Cancer Hospital and Institute, Beijing 100142, P.R. China
}

Received July 6, 2018; Accepted November 23, 2018

DOI: $10.3892 / \mathrm{ol} .2019 .9920$

\begin{abstract}
In the current era of multi-disciplinary treatment, precise and detailed diagnosis prior to treatment is crucial for clinical practice. For different lesions that fit different indications, the optimum approach for treatment differs significantly. Thus, the recent 8th American Joint Committee on Cancer classification system has introduced 'clinical stage' as a criterion. Endoscopic ultrasonography (EUS) has been the first-line choice for pretreatment staging; however, there is no standardization of the depth classification nor a standard EUS method. Additionally, the accuracy for this diagnostic test has ranged between $<40$ and $90 \%$ in previous studies. The aim of the present study was to determine the accuracy of EUS, identify the discrepancies between EUS and histological results, and analyze the underlying causes. Between June 2014 and February 2016, EUS was performed on gastric carcinoma specimens from 60 consecutive patients. EUS was performed on the resected specimens following surgery, but prior to fixation in formalin, invasion of the gastric wall was determined and the deepest location was marked with sutures. The ultrasound images were independently interpreted, and the quality of the images was scored by two endoscopists. Subsequently, the ultrasound images were compared with the pathological results of the same section. The overall accuracy of EUS was $75 \%$. For locally advanced gastric cancers, EUS had a relatively high accuracy $(33 / 43,86 \%)$. The EUS results
\end{abstract}

Correspondence to: Professor Jia-Fu Ji, Department of Gastrointestinal Surgery, Beijing Cancer Hospital, Key Laboratory of Carcinogenesis and Translational Research (Ministry of Education), Peking University Cancer Hospital and Institute, 52 Fucheng Road, Beijing 100142, P.R. China

E-mail: jijiafu@hsc.pku.edu.cn

*Contributed equally

Abbreviations: AJCC, American Joint Committee on Cancer; EUS, endoscopic ultrasound

Key words: gastric cancer, endoscopic ultrasound, staging, pathology corresponded well with the pathological hematoxylin and eosin staining results, and the deepest points determined by EUS were confirmed by pathology in the majority of cases $(85 \%)$. In total, 50 and 10 cases were scored as having high/moderate and low quality, associated with accuracies of $86 \%(43 / 50)$ and $20 \%(2 / 10)$, respectively. EUS is valuable for pretreatment T-staging, particularly for advanced cases. Proximal stomach cancer exhibited a tendency for improved accuracy. Overall, the results of the present study suggest that standardized scanning processes, particularly including all-encompassing scanning, proper probe-placement and high image quality, lead to improved accuracy of EUS.

\section{Introduction}

In the current era of multi-disciplinary treatment, precise and detailed diagnosis prior to treatment is crucial for clinical practice. In terms of gastric carcinoma, clinical staging is crucial for determining the optimal therapeutic strategy and is indispensable, since there will be no pretreatment histological staging evidence. Evaluation of the response to neoadjuvant therapy and determining the appropriate time to perform surgery also need precise clinical staging and evaluation. For early-stage cancer, decision making also depends on precise staging prior to endoscopic or surgical resection. The prognosis and selection of the appropriate treatment strategy depend markedly on the depth of invasion into the gastric wall. For different lesions, the optimum approach for treatment differs significantly. Thus, the recent 8th American Joint Committee on Cancer (AJCC) classification system has introduced the 'clinical stage' system $(1,2)$. Endoscopic ultrasonography (EUS) is recommended in the guidelines for pretreatment staging $(3,4)$.

EUS was first introduced into clinical practice in the 1980s (5) and has rapidly evolved into a reliable technique for the diagnosis of lesions of the digestive tract. In fact, the clear observation of the different layers of the gastric wall makes EUS one of the most valuable tools for T staging (6). However, there is no standardization of the depth classification nor a standard EUS method. There is also controversy regarding the diagnostic accuracy of EUS staging, with significant variability observed in previous studies (43-92\%) (7-10). In addition, a number of meta-analyses has also reported marked heterogeneity (11-15). 
Nevertheless, it remains unclear why a theoretically good tool yields such poor results and why such marked heterogeneity exists between different studies, and no study has yet analyzed the underlying causes of these discrepancies. Furthermore, EUS results have not been associated with pathology results, thus it is not clear where the problem lies.

The present prospective study was designed to analyze whether EUS is helpful for the staging of gastric carcinoma and to determine to what extent the accuracy may be improved. In the present study, EUS was performed on the resected specimen following surgery, prior to fixation in formalin, invasion of the gastric wall was evaluated and the deepest location was marked with sutures. Subsequently, the ultrasound images were compared with the pathological results to determine the accuracy of EUS staging, identify any discrepancy between the EUS and histological results, and analyze the underlying causes. To the best of our knowledge, the present study is the first in vitro study to investigate the accuracy of EUS for either early or advanced gastric carcinoma.

\section{Materials and methods}

EUS. Between June 2014 and February 2016, EUS was performed on gastric carcinoma specimens from 60 consecutive patients (33 men and 27 women). The patients ranged in age between 27 and 73 years (mean age, 56 years). Total or partial gastrectomy was performed in all patients, and a histopathological diagnosis was obtained for each patient. Surgery was the primary treatment; patients who had received any abdominal surgery or other antitumor therapy prior to gastrectomy were excluded (Fig. 1).

Post-operatively, each specimen was filled with physiological saline and placed in a container filled with physiological saline before fixation in formalin. To simulate the in vivo situation as far as possible, the specimens were not cut and remained as a lumen. EUS was performed on the resected specimen. Following resection, the specimen was filled with physiological saline and placed in a container filled with physiological saline prior to fixation in formalin (Fig. 2A and B). Blood clots and debris were cleaned first when required. The ultrasound probe (model EG-530UR; Fujifilm Corporation, Tokyo, Japan) moved from the distal to proximal side along the longitudinal axis of the stomach (Fig. 2C and D). The invasion of the gastric wall was carefully evaluated, and the location of the deepest tumor invasion was marked with sutures under real-time ultrasound image-guidance (Fig. 2E and F). The images were stored on a compact flash memory card. All EUS studies were performed by one endoscopist. The tumor-located gastric wall was spread evenly and fixed in formalin. Following fixation for $24 \mathrm{~h}$, the specimen underwent pathological examination following serial sectioning at an interval of 5-10 mm, during which the section marked with sutures was labeled and recorded.

Ethical approval was obtained from the Beijing Cancer Hospital Research Ethics Committee and the study was performed in accordance with The Declaration of Helsinki. All patients or their families provided written informed consent before undergoing any examination and treatment. The study was registered on clinicaltrials.gov (no. NCT02226224).

Data analysis. The EUS images were reviewed by two endoscopists with staging of the tumor in accordance with the AJCC staging system (8th edition) and assignment of a level of confidence to the interpretation. The images were independently interpreted without knowledge of the clinical characteristics or histopathological results. On EUS, the normal gastric wall may be separated into five layers. The first hyperechoic and second hypoechoic layers are recognized as the mucosa. The third hyperechoic layer is the submucosa. The fourth hypoechoic layer represents the muscularis propria, and the fifth hyperechoic layer is the subserosa and serosa. According to the AJCC 8th edition Tumor-Node-Metastasis staging system, the degree of tumor penetration into the gastric wall was categorized according to the deepest layer invaded, as follows: i) T1a, a dark expansion or thickening of layers 1 and 2 without interruption to the third layer corresponding to infiltration of the superficial and deep mucosa; ii) $\mathrm{T} 1 \mathrm{~b}$, the first to third hypoechoic layers with destruction of the normal structures corresponding to infiltration of the submucosa; iii) T2, a dark expansion of layers 1-4, representing penetration into the muscularis propria; iv) T3, layers 1-4 of the gastric wall cannot be distinguished and the hypoechoic area has an irregular outer border. These results indicate invasion of the subserosa; v) T4a, the whole hypoechoic area of the gastric wall is invaded and the bright line is interrupted; this represents invasion of the serosa; and (vi) T4b, extension of the mass into surrounding organs such as the liver, pancreas and spleen is staged as pT4b disease (16). However, T4b cases were not included in the present study.

The EUS image quality was scored on the basis of the detection repeatability, appropriate probe placement and clarity of the five gastric wall layers, including the lesion (17). The pathological diagnosis was made by two pathologists, and the marked section was assessed separately. The pathologists were not aware of the EUS results. Finally, the pathological diagnosis and EUS prediction were compared and whether the marked point was indeed the deepest point, as determined by the pathological results, was recorded. The histological section and the EUS imaging section of the marked point were compared. When there was a discrepancy between the pathological and ultrasound results, this was discussed, and the pathologists and endoscopists would reexamine their images.

In the statistical analysis, the continuous variable is described as the mean \pm standard deviation, and the categorical variable is described as the proportion. For determining the factors affecting consistency, a $\chi^{2}$ test was used. All statistical analyses were performed using Stata statistical software version 13.0 (StataCorp LP, College Station, TX, USA). $\mathrm{P}<0.05$ (two-tailed) was considered to indicate a statistically significant difference.

\section{Results}

The general clinicopathological characteristics of the study cohort are summarized in Table I. The diagnoses at histological examination included poorly differentiated adenocarcinoma (including signet-ring cell) $(n=33)$ and well-differentiated adenocarcinoma $(n=27)$. In total, 25 patients received total gastrectomy, whereas 35 received subtotal gastrectomy with lymphadenectomy (3 proximal and 32 distal). T-staging of 1, 2, 3 and 4 were assigned in 17, 14, 14 and 15 patients, respectively. 
Table I. Patient demographics and pathological stages.

\begin{tabular}{lcc}
\hline Variable & Value & Proportion, \% \\
\hline Mean age, years & $56 \pm 14$ & \\
Sex & & 55 \\
Male & 33 & 45 \\
Female & 27 & \\
pT & & 29 \\
T1 & 17 & 23 \\
T2 & 14 & 23 \\
T3 & 14 & 25 \\
T4a & 15 & \\
Histology & & 45 \\
Differentiated & 27 & 55 \\
Undifferentiated & 33 & \\
Lauren classification & & 48 \\
Intestinal type & 29 & 17 \\
Diffuse type & 10 & 35 \\
Mixed type & 21 & 28 \\
Location & & 54 \\
Upper third & 11 & \\
Middle third & 32 & \\
Lower third & & \\
\hline & & \\
\hline
\end{tabular}

$\mathrm{n}=60 . \mathrm{pT}$, pathological stage.

It was attempted to obtain qualified images for each case. However, for certain large lesions, it was difficult to clearly visualize the five layers of the normal gastric wall. Furthermore, for certain locations, such as the gastro-esophageal junction, it was difficult to maintain a proper distance between the probe and gastric wall. Accordingly, 50 cases were scored as having high or moderate quality, which was associated with improved accuracy $(43 / 50,86 \%)$, whereas 10 cases were scored as low quality, associated with poorer accuracy (2/10, 20\%). Higher image quality corresponded to higher confidence in terms of interpreting the results.

The accuracies of EUS are presented in Table II. The overall accuracy was $75 \%$. The accuracy was the highest in cases of T3 stage disease, at 93\%. On the other hand, for early-stage cases in the present study, the accuracy was low, at $<50 \%$.

The factors that may affect the accuracy, such as the depth of the tumor, histology, Lauren classification and location, were analyzed. The results indicated that tumors located in the upper third of the stomach tended to be predicted more accurately, as were tumors involving the full layer of the gastric wall (T3). Furthermore, undifferentiated adenocarcinomas and those of Lauren classification mixed type tended to have higher accuracy (Table III), but no statistical significance was observed. Except for T stage, no statistically significant differences were identified, possibly because of the limited sample size.

In the majority of cases $(51 / 60,85 \%)$, the EUS-deepest points were confirmed by the pathological results. However, in 9 cases, the point marked by EUS did not represent the location of deepest tumor invasion $(9 / 60,15 \%)$.
Table II. Accuracy of EUS for T staging.

\begin{tabular}{lrrrrr}
\hline & \multicolumn{4}{c}{ Pathological stage } & \\
\cline { 2 - 4 } EUS stage & T1 & T2 & T3 & T4 & Total (n) \\
\hline T1 & 8 & 1 & 0 & 1 & 10 \\
T2 & 8 & 12 & 0 & 1 & 21 \\
T3 & 1 & 1 & 13 & 1 & 16 \\
T4a & 0 & 0 & 1 & 12 & 13 \\
Total (n) & 17 & 14 & 14 & 15 & 60 \\
Overall accuracy, \% & 47 & 86 & 93 & 80 & 75 \\
\hline
\end{tabular}

Overall accuracy was defined as $\mathrm{uT}=\mathrm{pT}$ cases/total pT cases. EUS, endoscopic ultrasonography.

To attempt to reveal the underlying causes of incorrect predictions, the discrepancies between the EUS and pathological results were analyzed. In one case (case no. 4), tumor cells infiltrated the stomach without destruction of its layers (Fig. 3). The biological behavior of certain tumors makes anatomy-based staging tools impossible to predict, and recognition of this subgroup of tumors is particularly important in clinical practice. Misleading results, particularly under-staging, leads to inefficient treatment in this situation, therefore further attention is warranted.

In case no. 10 (Fig. 4), the normal layers were destroyed by ulcer. However, the tumor may originate from the ulcer margin rather than the base in certain cases. In such cases, EUS cannot distinguish the tumor from the ulcer, thus resulting in an incorrect prediction. This is common in ulcerated early-stage gastric cancers, particularly in larger lesions. Furthermore, in these cases, the deepest invasive points determined by EUS and pathology are more likely to differ. Slight or no thickening of the gastric wall may be a sign of a benign ulcer combined with early cancer.

\section{Discussion}

Pretreatment diagnosis based on the invasion depth of gastric cancer has become increasingly important. Particularly for advanced-stage cases, the prognosis and the selection of the appropriate treatment strategy depend on the pre-operative staging of the tumor. However, following administration of pre-operative treatments, the original staging of the tumor cannot be obtained by pathological analyses. Emphasis on clinical staging has increased in recent years, and obtaining a precise and reliable clinical staging is a challenging and important problem.

As EUS is able to visualize the different layers of the gastric wall as corresponding sonographic layers, it is generally considered the effective tool for T staging. However, in previous studies, the accuracy of EUS ranged between $<50$ and $>90 \%$ (7-10). In addition, a number of meta-analyses have also identified marked heterogeneities (11-15). Therefore, the validity of EUS for staging remains controversial. A literature review revealed significant heterogeneities between the studies, and it was identified that these previous studies typically 
Table III. Factors potentially related to accuracy.

\begin{tabular}{|c|c|c|c|c|}
\hline \multirow[b]{2}{*}{ Variable } & \multicolumn{2}{|c|}{ EUS accuracy, n (\%) } & \multirow[b]{2}{*}{ Total (n) } & \multirow[b]{2}{*}{ P-value } \\
\hline & Accurate & Inaccurate & & \\
\hline $\mathrm{pT}$ & & & & 0.022 \\
\hline $\mathrm{T} 1$ & $8(47)$ & $9(53)$ & 17 & \\
\hline $\mathrm{T} 2$ & $12(86)$ & $2(14)$ & 14 & \\
\hline $\mathrm{T} 3$ & $13(93)$ & $1(7)$ & 14 & \\
\hline $\mathrm{T} 4 \mathrm{a}$ & $12(80)$ & $3(20)$ & 15 & \\
\hline Histology & & & & 0.235 \\
\hline Differentiated & $18(67)$ & $9(33)$ & 27 & \\
\hline Undifferentiated & $27(82)$ & $6(18)$ & 33 & \\
\hline Lauren classification & & & & 0.347 \\
\hline Intestinal type & $20(69)$ & $9(31)$ & 29 & \\
\hline Diffuse type & $7(70)$ & $3(30)$ & 10 & \\
\hline Mixed type & $18(86)$ & $3(14)$ & 21 & \\
\hline Location & & & & 0.638 \\
\hline Upper third & $9(90)$ & $1(10)$ & 10 & \\
\hline Middle third & $14(78)$ & $4(22)$ & 18 & \\
\hline Lower third & $22(69)$ & $10(31)$ & 32 & \\
\hline Total & $45(75)$ & $15(25)$ & 60 & \\
\hline
\end{tabular}

pT, pathological stage.

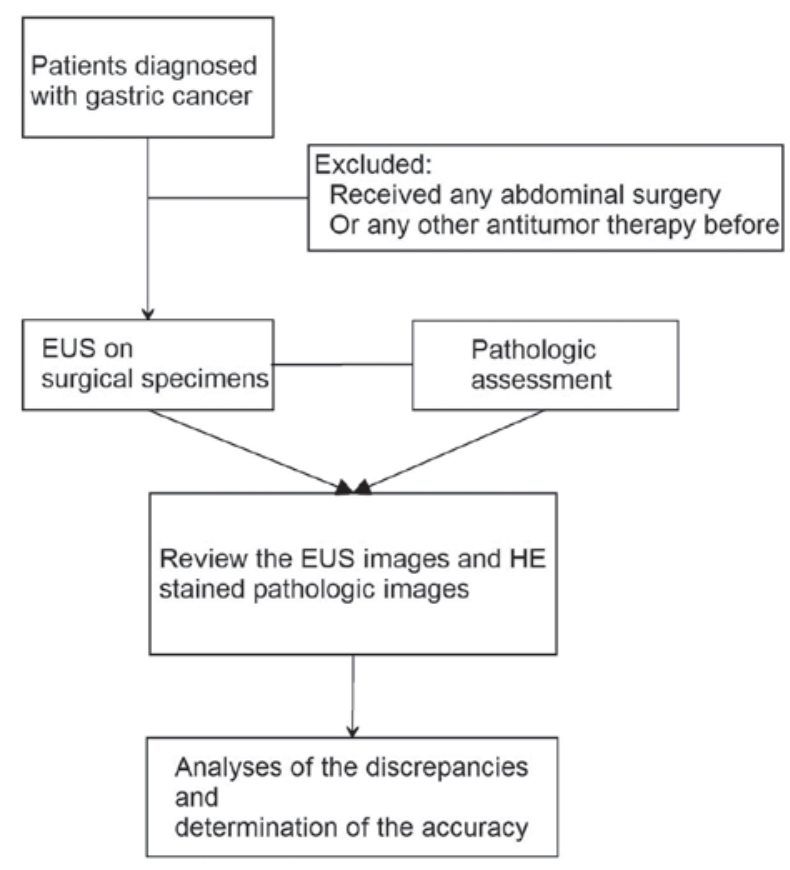

Figure 1. Study design. EUS, endoscopic ultrasonography; HE, hematoxylin and eosin.

focused on early-stage cancer. Furthermore, according to the latest AJCC staging system (8th edition) $(1,2)$, clinical staging must be made and EUS is essential for early- and advanced-stage cancer. Computed tomography scanning, as an alternative modality, has an even more marked heterogeneity in the literature (18).
As has been well-established, tumors are heterogenetic lesions. Different parts of a single tumor may have different depths of invasion. Furthermore, not all parts of a tumor are sectioned and interpreted during the pathological process in the majority of centers in China and other countries. In the majority of cases, the pathological results thus represent certain parts of the tumor. However, the EUS-determined deepest point may be located in a different part of the tumor, which may not be examined during the pathological process. Under these circumstances, the interpretation of the EUS images cannot be revised according to the pathological results, because they may be completely different. For these reasons, the present study was designed in which EUS was performed on resected specimens. By marking the point of interest, the association between the pathology and EUS results could be analyzed. This thus allowed revision of our understanding of EUS images in order to achieve improved accuracy. At the same time, the accuracy of EUS evaluation for tumor depth may be determined.

In the present study, following thorough EUS scanning, the tumors were serially sectioned. The pathologist judged and recorded whether the deepest point marking made under EUS guidance was indeed the deepest invasion part pathologically.

In the present study, the overall accuracy was $75 \%$. For locally advanced gastric cancers, EUS exhibited a relatively high degree of accuracy $(33 / 43,86 \%)$, whereas, for early-stage cases, the accuracy was low, at $<50 \%$. However, it should be noted that substantial bias existed in the present study, as only patients who underwent surgery were enrolled. The majority of cases diagnosed as early-stage gastric cancer would undergo endoscopic submucosal resection as the primary treatment in 
A

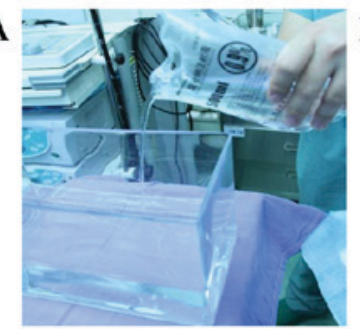

$\mathbf{E}$

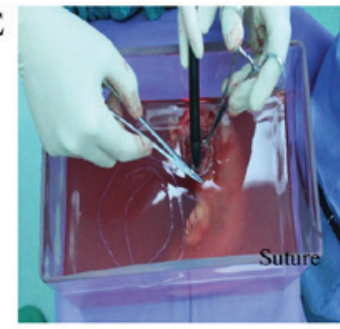

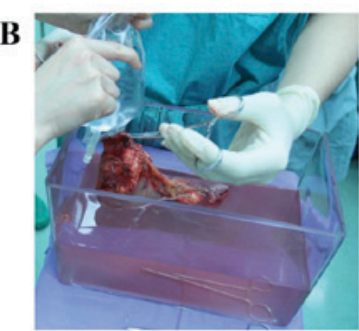
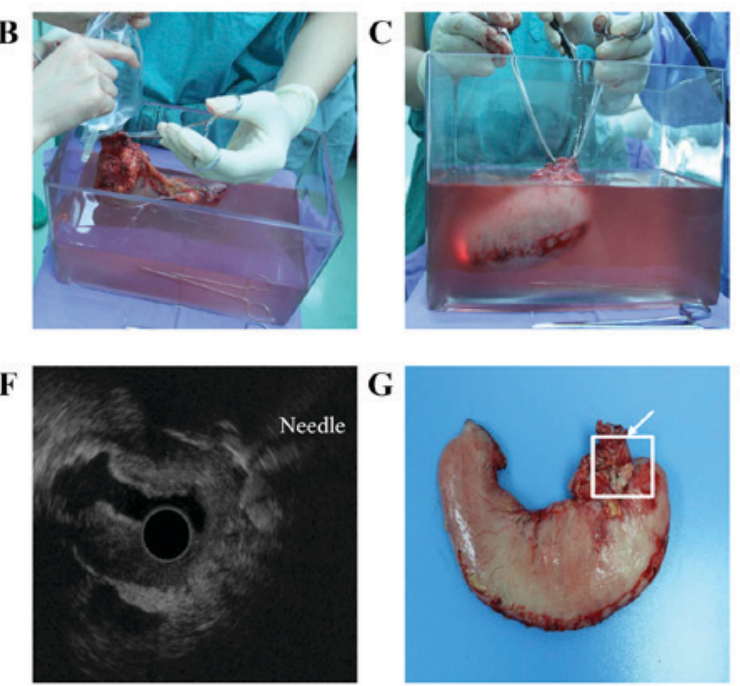

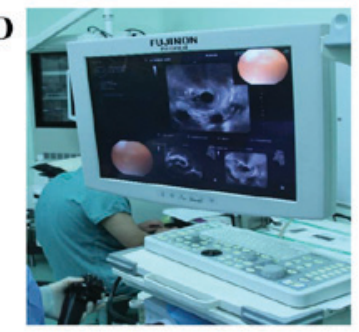

H

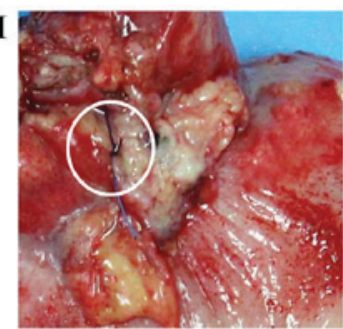

Figure 2. EUS procedure. (A) Fill the container with physiological saline, (B) The specimen was put in the container prior to fixation in formalin. (C) Endoscopic ultrasonography was performed on the resected specimen. (D) The invasion of the gastric wall was determined, and (E) The location of the deepest invasion was marked with suture. (F) Real-time guided marking by EUS. (G) The specimen after marking. (H) The suture on the specimen.

$\mathbf{A}$

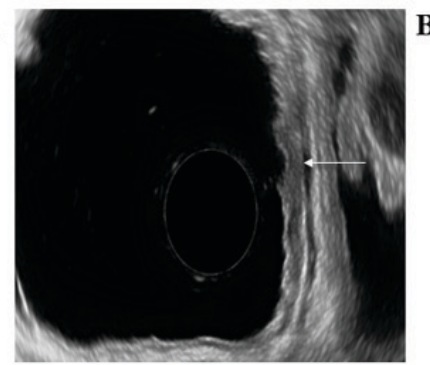

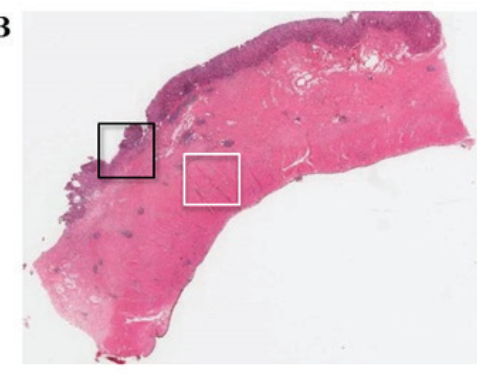
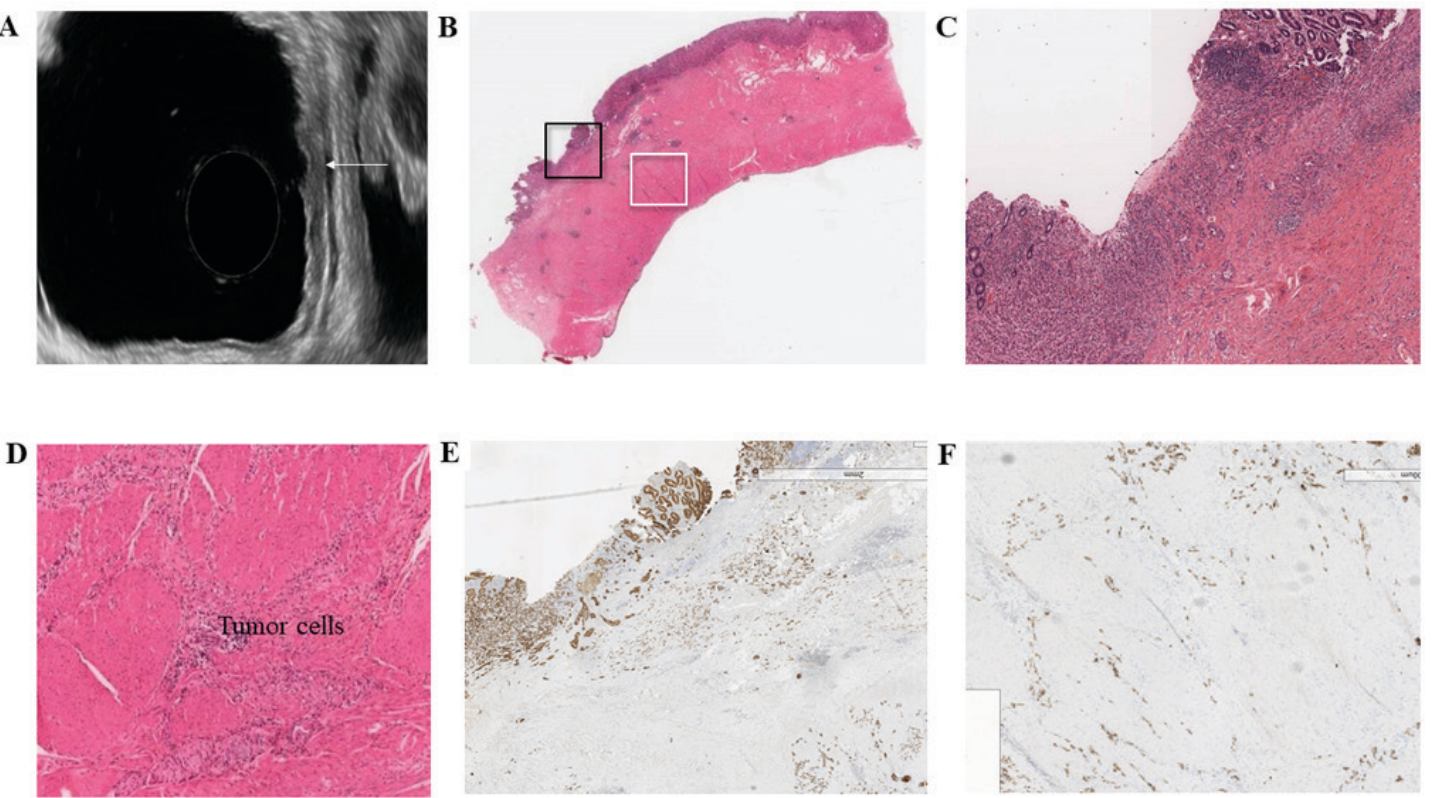

Figure 3. Results from an under-staged case of gastric cancer. (A) Endoscopic ultrasonographic image. The tumor presented as a slightly depressed superficial lesion invading into the third layer of the stomach. (B) Low-magnification view with hematoxylin and eosin staining. Darker cells with hyperchromatic nuclei were prominent in the mucosa and submucosa. High-magnification view of areas indicated by the (C) black box and (D) white box in (B). Tumor cells with hyperchromatic nuclei were identified to infiltrate the interfascicular portion of the muscularis propria, and continue into the serosa. Images of cytokeratin staining, with (E) and (F) corresponding to (C) and (D), respectively, clearly indicating that the tumor extends from the mucosa to the serosa.

our center, so the early-stage cases referred to surgery were thus likely to be accompanied by severe ulcers or were characterized by large or poorly differentiated tumors. Tumors with these features are already problematic for clinical evaluation of invasion using standard methods (19). The frequency and quality of EUS may be another reason for the low accuracy, because mini-probes were not applied.

Nevertheless, the EUS results corresponded well to the pathological hematoxylin and eosin staining results, and the deepest point determined by EUS was confirmed by pathology in the majority of cases (85\%), indicating that EUS is a valuable tool for pretreatment T-staging, particularly for advanced-stage cases and proximal stomach cancer, which exhibited a tendency for improved accuracy. It should be noted that the histology, Lauren classification and tumor depth may have confounding effects, as reported previously (20-22). The accuracy for lesions of different histology classification, i.e. Lauren classification or tumor depth may differ. However, owing to the limited sample size of the present study, no significant difference was identified in the present study.

Taken together, the results of the present study suggest that the use of a standardized scanning process and high-quality 

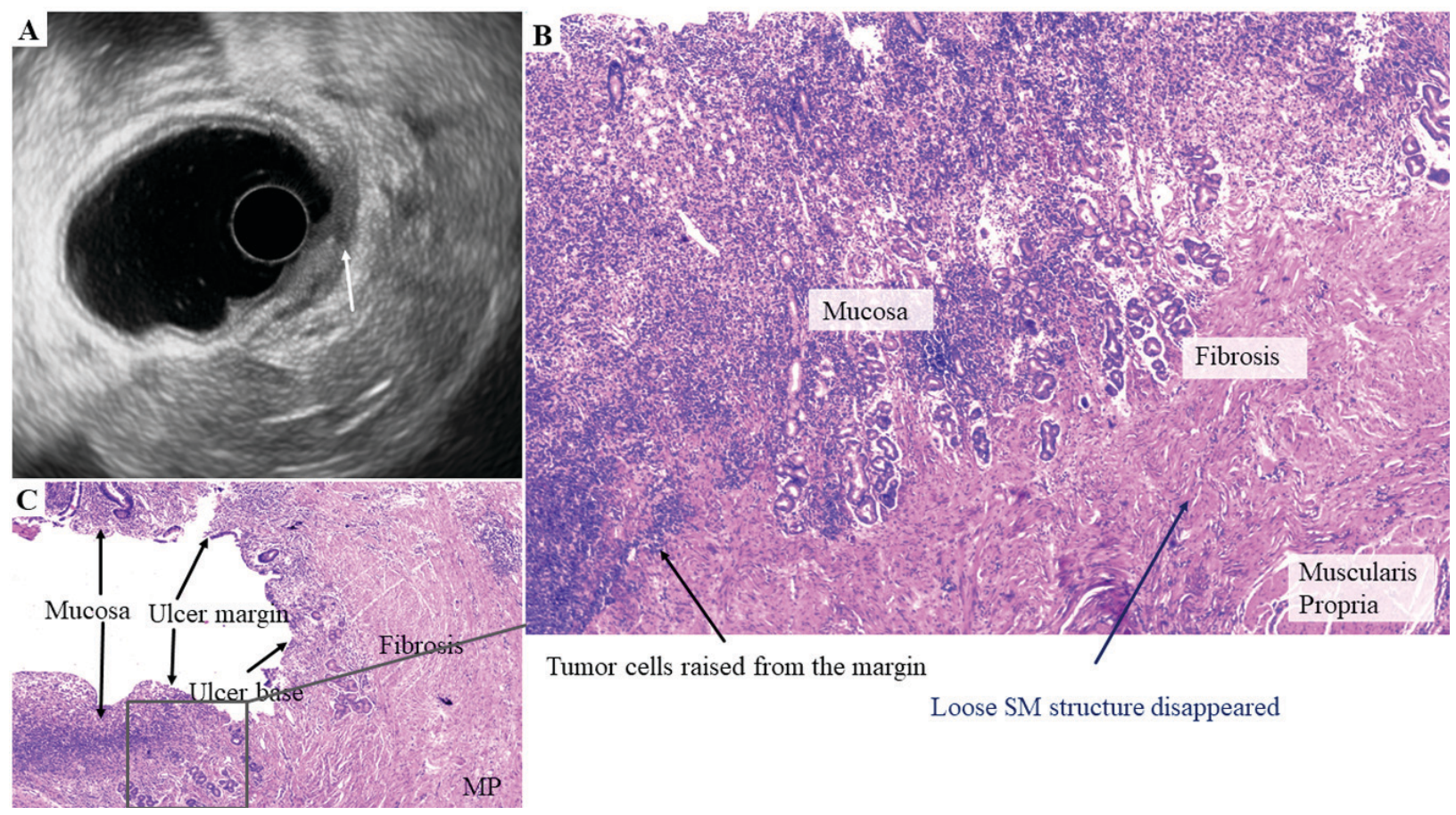

Loose SM structure disappeared

Figure 4. Results from an over-staged case. (A) Endoscopic ultrasonographic image. A depressed hypoechoic area extending to the fourth layer was observed. (B) Low-magnification view with hematoxylin and eosin staining. The loose SM layer disappeared, whereas prominent fibrosis pulling the MP up next to the mucosa was observed. (C) High-magnification view with hematoxylin and eosin staining of the area indicated by the grey box in (B). The cancer cells were identified to originate from the margin of the ulcer. SM, submucosal; MP, muscularis propria.

images would raise the accuracy of EUS. In particular, cleaning the debris of a large ulcer, all-encompassing scanning, proper probe-placement and high image quality are associated with improved accuracy.

The present study had certain limitations. The number of cases investigated was limited and the study was performed in vitro, and therefore it was not possible to fully simulate the various situations that may occur in clinical practice.

EUS was identified to be a reliable tool for pretreatment $\mathrm{T}$-staging with a satisfying accuracy, particularly for the advanced-stage tumors and proximal stomach cancers. Accordingly, creating standardized guidelines for EUS scanning and establishing criteria to ensure high image quality are expected to improve the accuracy of EUS staging.

\section{Acknowledgements}

The authors would like to thank Dr Zhong-Wu Li and Dr Yu Sun (Department of Pathology, Beijing Cancer Hospital, Key Laboratory of Carcinogenesis and Translational Research (Ministry of Education) and Peking University Cancer Hospital and Institute, (Beijing, China) for their help and instructions as pathologists. The authors would also like to thank Dr Lei Tang (Department of Radiology, Beijing Cancer Hospital, Key Laboratory of Carcinogenesis and Translational Research (Ministry of Education), Peking University Cancer Hospital and Institute, (Beijing, China) for advice.

\section{Funding}

The present study was supported by Beijing Municipal Science \& Technology Commission (grant no. Z171100001017135).
The study was also supported by Outstanding Key Person Funds from the Organization Department of the Beijing Municipal Committee (grant no. 2016000021469G188).

\section{Availability of data and materials}

The datasets used and analyzed during the present study are available from the corresponding author on reasonable request.

\section{Authors' contributions}

JFJ, YY and QW made essential contributions to the conception of the study. JFJ designed and supervised the study. QW and YY performed the study, collected the data and conducted the statistical analysis. QW, YY, ZYL and ZDB participated in the enrollment of patients and the interpretation of the results. YY drafted the paper. JJ, QW, ZYL and ZDB revised the paper for critical points. All authors read and revised the paper before final approval.

\section{Ethics approval and consent to participate}

Ethical approval was obtained from the Beijing Cancer Hospital Research Ethics Committee in 2014 (2014KT11) and the study was carried out in accordance with The Declaration of Helsinki. All patients or their families provided written informed consent before undergoing any examination and treatment. The study was registered on clinicaltrials.gov (no. NCT02226224).

\section{Patient consent for publication}

Not applicable. 


\section{Competing interests}

The authors declare that they have no competing interests.

\section{References}

1. Brierley JD, Gospodarwicz MK, Wittekind C and Amin MB: TNM classification of maligant tumours, 8th ed. Oxford: Wiley Blackwell, 2017.

2. Amin MB, Edge SB, Greene FL, Byrd DR, Brookland RK, Washington MK, Gershenwald JE, Compton CC, Hess KR, Sullivan DC, et al: AJCC cancer staging manual, 8th edition. New York: Springer, 2017.

3. Smyth EC, Verheij M, Allum W, Cunningham D, Cervantes A and Arnold D; ESMO Guidelines Committee: Gastric Cancer: ESMO Clinical Practice Guidelines for diagnosis, treatment and follow-up. Ann Oncol 27 (Suppl 5): v38-v49, 2016.

4. Japanese Gastric Cancer Association: Japanese gastric cancer treatment guidelines 2014 (ver. 4). Gastric Cancer 20: 1-19, 2017

5. Heyder N, Kaarmann H and Giedl J: Experimental investigations into the possibility of differentiating early from invasive carcinoma of the stomach by means of ultrasound. Endoscopy 19 228-232, 1987.

6. Papanikolaou IS, Triantafyllou M, Triantafyllou K and Rösch T: EUS in the management of gastric cancer. Ann Gastroenterol 24 9-15, 2011.

7. Kutup A, Vashist YK, Groth S, Vettorazzi E, Yekebas EF, Soehendra N and Izbicki JR: Endoscopic ultrasound staging in gastric cancer: Does it help management decisions in the era of neoadjuvant treatment? Endoscopy 44: 572-576, 2012.

8. Jurgensen C, Brand J, Nothnagel M, Arlt A, Neser F, Habeck JO, Schreiber S, Stölzel U, Zeitz M and Hampe J: Prognostic relevance of gastric cancer staging by endoscopic ultrasound. Surg Endosc 27: 1124-1129, 2013.

9. Lee HH, Lim CH, Park JM, Cho YK, Song KY, Jeon HM and Park CH: Low accuracy of endoscopic ultrasonography for detailed T staging in gastric cancer. World J Surg Oncol 10: 190, 2012.

10. Yoshinaga S, Oda I, Nonaka S, Kushima R and Saito Y: Endoscopic ultrasound using ultrasound probes for the diagnosis of early esophageal and gastric cancers. World J Gastrointest Endosc 4: 218-226, 2012

11. Kwee RM and Kwee TC: The accuracy of endoscopic ultrasonography in differentiating mucosal from deeper gastric cancer. Am J Gastroenterol 103: 1801-1809, 2008.

12. Cardoso R, Coburn N, Seevaratnam R, Sutradhar R, Lourenco LG, Mahar A, Law C, Yong E and Tinmouth J: A systematic review and meta-analysis of the utility of EUS for preoperative staging for gastric cancer. Gastric Cancer 15 (Suppl 1): S19-S26, 2012.
13. Puli SR, Batapati Krishna Reddy J, Bechtold ML, Antillon MR and Ibdah JA: How good is endoscopic ultrasound for TNM staging of gastric cancers? A meta-analysis and systematic review. World J Gastroenterol 14: 4011-4019, 2008.

14. Mocellin S, Marchet A and Nitti D: EUS for the staging of gastric cancer: A meta-analysis. Gastrointest Endosc 73: 1122-1134, 2011.

15. Mocellin S and Pasquali S: Diagnostic accuracy of endoscopic ultrasonography (EUS) for the preoperative locoregional staging of primary gastric cancer. Cochrane Database Syst Rev: CD009944, 2015.

16. Ajani JA, D'Amico TA, Almhanna K, Bentrem DJ, Chao J, Das P, Denlinger CS, Fanta P, Farjah F, Fuchs CS, et al: Gastric cancer, version 3.2016, NCCN Clinical Practice Guidelines in Oncology. J Natl Compr Canc Netw 14: 1286-1312, 2016.

17. Yamamoto S, Nishida T, Kato M, Inoue T, Hayashi Y, Kondo J, Akasaka T, Yamada T, Shinzaki S, Iijima H, et al: Evaluation of endoscopic ultrasound image quality is necessary in endosonographic assessment of early gastric cancer invasion depth. Gastroenterol Res Pract 2012: 194530, 2012.

18. Seevaratnam R, Cardoso R, McGregor C, Lourenco L, Mahar A, Sutradhar R, Law C, Paszat L and Coburn N: How useful is preoperative imaging for tumor, node, metastasis (TNM) staging of gastric cancer? A meta-analysis. Gastric Cancer 15 (Suppl 1): S3-S18, 2012

19. Park JS, Kim H, Bang B, Kwon K and Shin Y: Accuracy of endoscopic ultrasonography for diagnosing ulcerative early gastric cancers. Medicine (Baltimore) 95: e3955, 2016.

20. Tsuzuki T, Okada H, Kawahara Y, Nasu J, Takenaka R, Inoue M, Kawano S, Kita M, Hori K and Yamamoto K: Usefulness and problems of endoscopic ultrasonography in prediction of the depth of tumor invasion in early gastric cancer. Acta Med Okayama 65: 105-112, 2011.

21. Park YS, Lee D, Lee DH, Kim NY, Jeong SH, Kim JW, Hwang JH, Lee SH, Kim JS, Jung HC and Song IS: Assessment of factors affecting the accuracy of endoscopic ultrasonography in T2 stage gastric cancer. Korean J Gastroenterol 52: 86-90, 2008 (In Korean).

22. Han C, Lin R, Shi H, Liu J, Qian W, Ding Z and Hou X: The role of endoscopic ultrasound on the preoperative $T$ staging of gastric cancer: A retrospective study. Medicine (Baltimore) 95: e4580, 2016.

This work is licensed under a Creative Commons Attribution-NonCommercial-NoDerivatives 4.0 International (CC BY-NC-ND 4.0) License. 